Reprod. Nutr. Dévelop., 1981, 21 (5A), 661-670.

\title{
Influence du mode d'élevage sur le comportement et I'activité hypophyso-corticosurrénalienne du porcelet
}

\author{
par R. DANTZER ( $\left.{ }^{1}\right)$, P. MORMÈDE $\left(^{(}\right)$ \\ avec la collaboration technique de R. M. BLUTHÉ
}

Groupe de Recherches sur le Stress, I.N.R.A., 180, chemin de Tournefeuille, 31300 Toulouse.

Summary. Influence of weaning time on piglef behaviour and pituitary-adrenal reactivity.

The pituitary-adrenal activity and behavioural reactions of 3-week weaned piglets kept in flat-decks were compared with those of 6-week weaned piglets kept in the maternity pen.

Early weaning induced a transitory increase of plasma corticosteroid levels, while 6-week weaning did not influence those levels. Lower plasma corticosteroid levels were observed in piglets kept with the sow during post-natal weeks 4 and 5 .

To assess whether early weaning would affect subsequent reactivity, both groups of piglets were individually submitted to an open-field test, a neophobia test and a continuous avoidance schedule when they were 7-9 weeks old. Early-weaned piglets reacted less to the open field test, but pituitary-adrenal response was the same in both experimental groups. Early-weaned piglets were slower in approaching a new object (a food container) in a familiar environment (neophobia test), suggesting that they were more neophobic than 6-week weaned piglets. Avoidance conditioning performances were different in the two sexes : females had the same score whatever their previous rearing conditions, while 6-week weaned castrated males were relatively deficient in learning the task.

Taken as a whole, the behavioural and pituitary-adrenal differences observed in the present study do not indicate that early weaning in flat-deck has long-term detrimental effects on the adaptive abilities of pigs.

\section{Introduction.}

Pour une même production animale comme le porcelet, diverses techniques d'élevage sont possibles, avec tous les intermédiaires entre le système traditionnel (sevrage à 6-8 semaines) et le sevrage très précoce à 3-4 jours. Le choix d'une technique est habituellement basé sur des considérations économiques et zootechniques. Or les conditions de vie des animaux et, partant, leur expérience peuvent être très différentes suivant le mode d'élevage retenu : la plupart des techniques de sevrage précoce imposent à l'animal, outre la rupture des relations mère-jeune, un véritable environnement appauvri caractérisé par un faible niveau de stimulation et la limitation de l'espace disponible par animal.

(1) Adresse actuelle : Laboratoire de Neurobiologie des Comportements, Université Bordeaux II, 146, rue Léo-Saignat, 33076 Bordeaux Cedex. 
De nombreuses expériences réalisées sur les animaux de laboratoire ont démontré que le milieu psychosocial dans lequel évolue l'individu de même que le niveau de stimulation auquel il est soumis exercent des effets à terme particulièrement importants lorsque l'action du milieu se situe au moment du développement. Ainsi le niveau de stimulation au cours de la période post-natale influence profondément le comportement ultérieur, les réponses endocriniennes ef la résistance physique aux agressions (Denenberg, 1969).

Compte tenu de ces données et des problèmes zootechniques et pathologiques rencontrés en élevage industriel (Dantzer et Mormède, 1979), on est en droit de se demander si les nouvelles conditions d'élevage imposées aux jeunes animaux ne fragilisent pas l'organisme et ne le rendent pas moins capable de s'adapter aux agressions.

Pour répondre à cette question, il est nécessaire de pouvoir apprécier les capacités d'adaptation de sujets de même origine génétique placés dans des milieux différents. La combinaison de mesures d'activité neuroendocrinienne ef d'observations comportementales dans des épreuves standardisées (« open-field», évitement) fournit une estimation des capacités d'adaptation et nous avons montré récemment que de tels critères permettaient de typer des porcs d'origine génétique différente (Dantzer et Mormède, 1978 ; Mormède et Dantzer, 1978). Nous nous proposons de rechercher ici si la même approche est capable de révéler des différences entre des porcelets sevrés à 6 semaines dans la loge de maternité et des animaux sevrés à 3 semaines et élevés en cage grillagée.

\section{Méthodes.}

\section{Animaux ef conditions d'élevage.}

Quatre truies gestantes pluripares (Camborough saillies par verrat Landrace) ont été rentrées simultanément au laboratoire un mois avant terme et maintenues à l'attache dans une loge de maternité $(1,75 \times 2,60 \mathrm{~m})$. La nourriture, sous forme de farine, éłait distribuée en deux repas, à raison de 5-6 kg par truie, sauf le samedi et le dimanche où un seul repas était donné par jour. L'eau était disponible à volonté. Un rationnement a été imposé dans la dernière semaine de gestation, en même temps que de la paille était fournie. Les mises bas ont eu lieu fin février, avec un intervalle de 6 jours entre la première et la dernière. Quarante et un porcelets ont été obtenus (1414-11-2), dont 7 sont morts par écrasement dans les 4 premiers jours suivant la misebas. Six porcelets ont été transférés à la truie n'ayant eu que 2 porcelets le lendemain de la mise bas. Les dents et la queue des porcelets ont été coupées le jour de la naissance et les mâles castrés à 2 semaines. La paille était renouvelée tous les jours. Un abreuvoir automatique et un distributeur d'aliments en granulés (aliment de premier âge, à 18 p. 100 de matières protéiques brutes) ont été rajoutés dans chaque loge au $10^{\mathrm{e}}$ jour. La température ambiante était maintenue à $18-20^{\circ} \mathrm{C}$ et des lampes radiantes assuraient un chauffage d'appoint pour les porcelets.

Deux portées ont été sevrées à 3 semaines : 17 porcelets ont été regroupés par taille ef transférés dans deux cages grillagées (« flat-deck ») adjacentes (1,20 × 1,20 m ; plancher en maille de $10 \mathrm{~mm}$ de côté, fil de $2 \mathrm{~mm}$ de diamètre) assurant une surface au sol de 0,16-0,18 $\mathrm{m}^{2}$ par animal. La nourriture (aliment de premier âge de la $3^{\mathrm{e}}$ à la $4^{\mathrm{e}}$ semaine, aliment de sevrage à 18 p. 100 de matières protéiques brutes jusqu'à 
6 semaines, «Porcelet démarrage » à 14 p. 100 de matières protéiques brutes par la suite) était disponible à volonté dans une auge occupant la face avant de la cage et permettant l'accès simultané de 6 animaux; chaque cage était munie d'un abreuvoir automatique. Les cages étaient placées dans une pièce de $6,25 \times 4,4 \mathrm{~m}$, maintenue à $22-24^{\circ} \mathrm{C}$, ventilée, et faiblement éclairée par une ampoule de $25 \mathrm{~W}$ en $220 \mathrm{~V}$. L'espace disponible pour les porcelets laissés avec la truie a été dédoublé en permettant l'accès des animaux à la loge contiguë à 3 semaines et demie. L'aliment était le même que celui distribué en cage et la paille était renouvelée tous les jours après nettoyage des loges. Le sevrage de ces animaux a été réalisé à 6 semaines, en retirant la truie.

A 7 semaines et demie, l'aliment a été rationné pour les deux séries d'animaux à 5 p. 100 du poids vif et la température abaissée à $20^{\circ} \mathrm{C}$ dans la pièce contenant les cages grillagées. Les lots ont été réduits à 6 animaux par cage à la $8^{e}$ semaine $\left(0,22 \mathrm{~m}^{2} \mathrm{de}\right.$ surface au sol par animal).

Déroulement des observations.

1) Taux de base des corticostéroïdes plasmatiques. - Le point de départ des observations est représenté par le jour du sevrage précoce (J0). Pour rechercher l'influence du moment du sevrage et du mode d'élevage sur l'activité hypophyso-cortico surrénalienne, du sang a été prélevé sur héparine $(10 \mu \mathrm{l} / \mathrm{ml}$ de sang) par ponction de la veine cave antérieure sur 6 animaux choisis au hasard dans chaque groupe expérimental, avant et après le sevrage ef tout au long du post-sevrage, le matin entre $8 \mathrm{~h} 30$ et $9 \mathrm{~h}$ $(\mathrm{J} 0, \mathrm{~J} 1, \mathrm{J4}, \mathrm{J} 7, \mathrm{~J} 14, \mathrm{~J} 22, \mathrm{~J} 23, \mathrm{~J} 25, \mathrm{~J} 29$ et J30, J22 représentant le jour du sevrage à 6 semaines). Après centrifugation des prélèvements, le plasma a été congelé $\left(-20^{\circ} \mathrm{C}\right)$. Les corticostéroïdes plasmatiques ont été dosés ultérieurement par radio-compétition protéique (Mormède et Dantzer, 1978).

2) Test d'open-field et de néophobie. - Un test de réactivité à un environnement nouveau et à la privation sociale (open field) (Dantzer, 1979) a été réalisé à 7 semaines (J29, et J30) sur 16 animaux, à raison de 4 mâles castrés et de 4 femelles par groupe expérimental. Le nombre de vocalisations, de défécations et de fuites, l'activité locomotrice, le temps passé couché et debout sans bouger ont été décomptés au cours d'une séance de 15 min pour chaque animal, par un observateur ignorant l'origine des porcelets. En fin de séance, une note de réactivité a été attribuée d'après le comportement observé $(0=$ sédation, 1 = réactivité diminuée, $2=$ réactivité normale, $3=$ réactivité augmentée, 4 = excitation intense) (Dantzer, 1979). Une prise de sang à la veine cave antérieure a été faite juste avant la séance et à la fin de celle-ci pour la mesure des taux d'ACTH par radioimmunologie (Kit ACTHK-CEA, Mormède et Dantzer, 1978) ef des corticostéroïdes plasmatiques. Vingt-quatre à 48 h après la séance d'observation, mais dans tous les cas après 24 h de jeûne, la réaction de néophobie vis-à-vis d'un récipient métallique cylindrique contenant de la nourriture et placé dans l'environnement précédemment exploré (Dantzer et Mormède, 1978) a été déterminée, sưr les mêmes animaux, par mesure de la latence d'approche du récipient et de la latence d'alimentation, au cours d'une séance de $10 \mathrm{~min} .\left(^{*}\right)$.

${ }^{*}$ ) La latence d'approche est le temps mis par l'animal, après introduction dans l'enceinte-test pour flairer avec le groin le récipient de nourrifure; la latence d'alimentation est le temps mis pour commencer à manger. 
3) Test d'évitement. - A 8-9 semaines, 22 animaux ont été soumis, en deux séries consécutives sur 5 jours chacune, avec un intervalle de deux jours entre les séries, à l'apprentissage d'un évitement continu (Dantzer et Mormède, 1978) : après une séance d'exploration de la cage à deux compartiments, les animaux sont soumis à 4 séances de conditionnement au cours desquelles ils doivent apprendre à éviter des chocs électriques $(26 \mathrm{~mA}, 0,25 \mathrm{~s})$ programmés toutes les $10 \mathrm{sec}$, en passant d'un côté à l'autre de la cage à deux compartiments, cette réponse retardant l'arrivée du prochain choc d'un délai de $30 \mathrm{sec}$. Les séances ont eu lieu le matin, entre $8 \mathrm{~h}$ et $11 \mathrm{~h}$, avant la distribution de nourriture.

\section{Résultats.}

Le sevrage à 3 semaines n'a eu de conséquences ni sur la croissance des animaux (le poids à 6 semaines est le même dans les deux groupes : 13,9 $\pm 0,73 \mathrm{~kg}$ contre $14,1 \pm 0,58 \mathrm{~kg}$ pour les animaux sevrés à 6 semaines et à 3 semaines respectivement, $\left.\mathrm{F}_{31}^{1}=0,04\right)$, ni sur leur état de santé.

1) Taux de base des corticostéroïdes plasmatiques. - La figure 1 illustre l'évolution des faux de corticostéroïdes plasmatiques entre la $3^{\mathrm{e}}$ et la $7^{\mathrm{e}}$ semaine chez les porcelets sevrés à 3 semaines et chez ceux sevrés à 6 semaines. Une analyse de variance à 2 facteurs ( 9 périodes $\times 2$ traitements, 6 répétitions) sur les transformés logarithmiques des taux plasmatiques de corticostéroïdes met en évidence un effet significatif des deux facteurs principaux (Période : $\mathrm{F}_{90}^{8}=5,64 ; \mathrm{P}<0,001$; traitement $\mathrm{F}_{90}^{1}=32,29$; $P<0,001)$, tandis que l'interaction période $\times$ traitement est proche de la signification $\left(\mathrm{F}_{90}^{8}=1,96 ; \mathrm{P}<0,10\right)$. L'évolution des taux plasmatiques de corticoïdes n'est donc

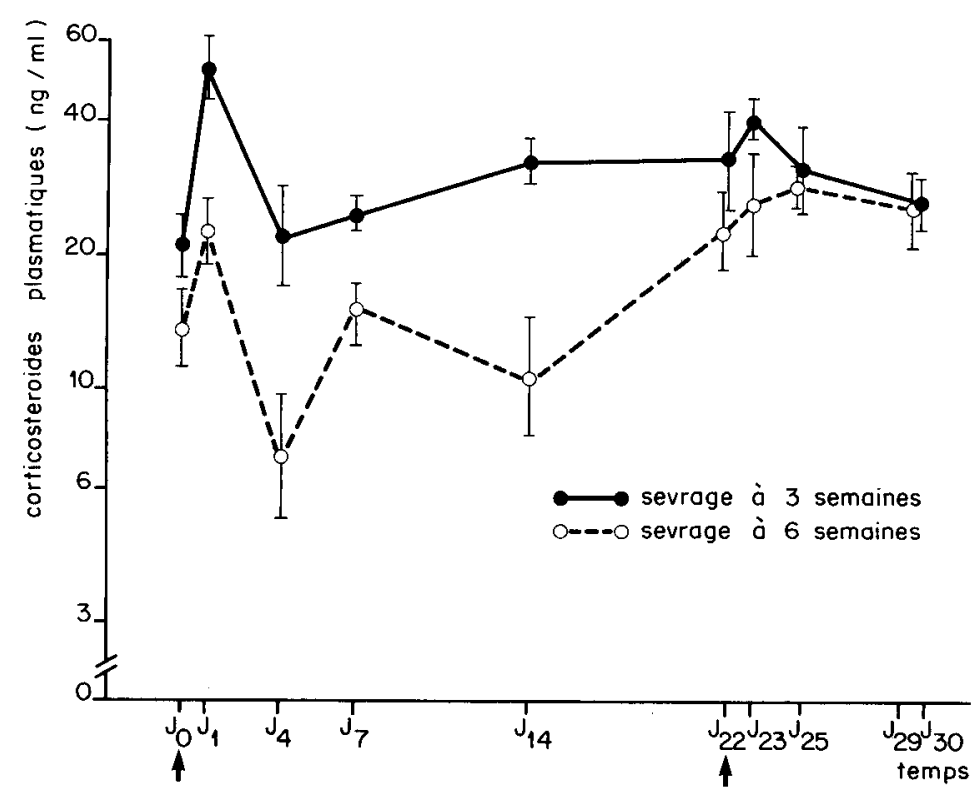

FIG. 1. - Evolution des taux de corticostéroïdes plasmatiques (moyenne \pm écart-type de la moyenne, $\mathrm{n}=6$ ) chez les porcelets sevrés (flèche) à 3 semaines (J0) et à 6 semaines (J22). 


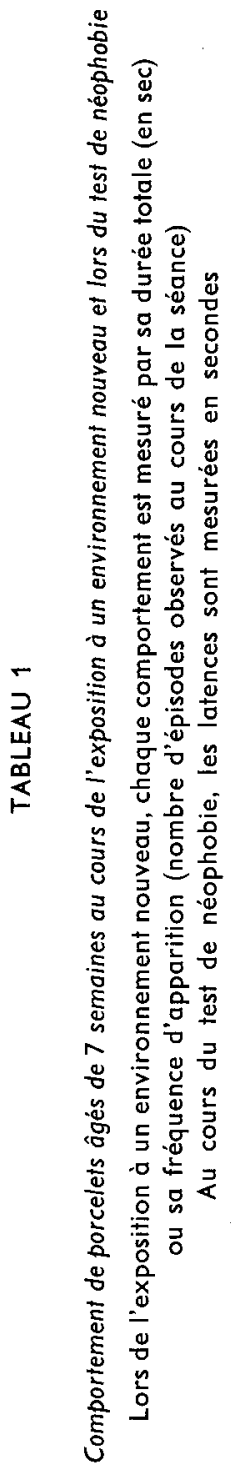

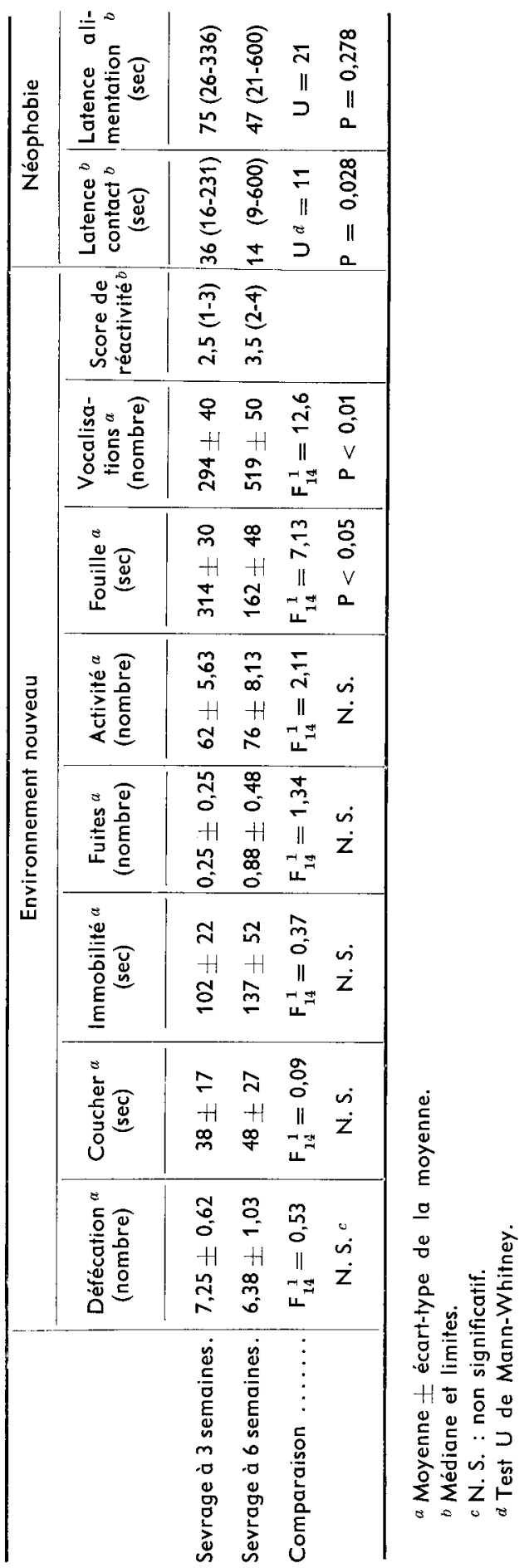


pas la même dans les deux groupes : la comparaison des moyennes deux à deux par le test de Newman-Keuls montre que chez les animaux sevrés précocément, les corticostéroïdes plasmatiques augmentent le lendemain de la manipulation $(P<0,10)$; par contre chez les animaux laissés avec la truie, une chute importante des corticostéroïdes plasmatiques apparaît au $4 \mathrm{e}$ jour et au $14 \mathrm{e}$ jour. Chez ces animaux, l'enlèvement de la truie au $22 \mathrm{e}$ jour n'affecte pas l'activité corticosurrénalienne.

2) Test d'open-field ef de néophobie. - Les animaux sevrés à 6 semaines présentent une réactivité plus importante au test de privation sociale que les porcelets sevrés à 3 semaines : le nombre de vocalisations émises est plus élevé ainsi que le score de réactivité (tabl. 1). Les animaux sevrés précocément passent deux fois plus de temps à fouiller la sciure répandue sur le sol que les animaux sevrés à 6 semaines. Ce comportement est corrélé négativement au nombre de vocalisations $(r=-0,52$, d.d.l. $=14$, $P<0,05$ ). Les autres comportements observés (activité, défécation, temps passé couché, temps passé debout, fuites) ne diffèrent pas suivant le mode d'élevage. Une analyse de variance à 2 facteurs (temps de prélèvements $\times$ date du sevrage, 8 répétitions) sur les transformés logarithmiques des taux plasmatiques de corticostéroïdes montre une augmentation des taux à la fin de la séance $(66,7 \mathrm{ng} / \mathrm{ml}$ contre $27,5 \mathrm{ng} / \mathrm{ml}$, $\left.F_{28}^{1}=34,93 ; P<0,001\right)$, mais pas d'effet du mode d'élevage $\left(F_{28}^{1}=0,03\right)$. Les taux d'ACTH sont également plus élevés en fin de séance $(342 \mathrm{pg} / \mathrm{ml}$ contre $58 \mathrm{pg} / \mathrm{ml}$ $\left.F_{28}^{1}=117,6 ; P<0,001\right)$ et ne sont pas modifiés par le mode d'élevage $\left(F_{28}^{1}=0,21\right)$ (fig. 2). Dans le test de néophobie, les animaux sevrés précocément mettent plus long-

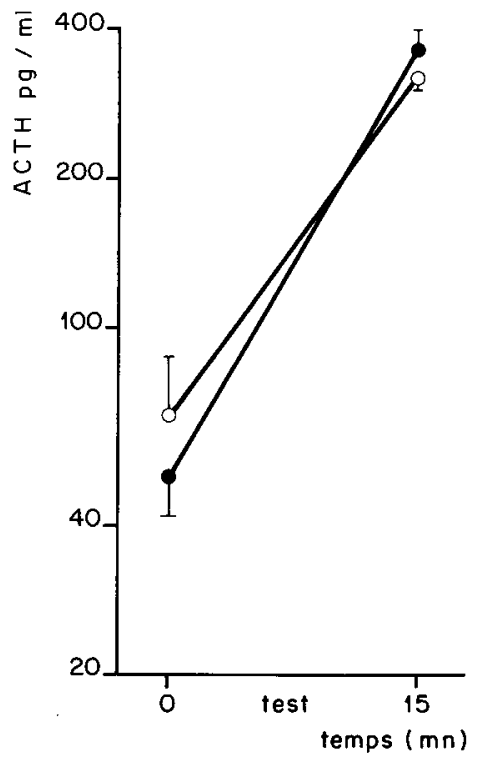

$\mathrm{ACTH}$
O Sevrage précoce (S 3)

- Sevrage traditionnel (S6)

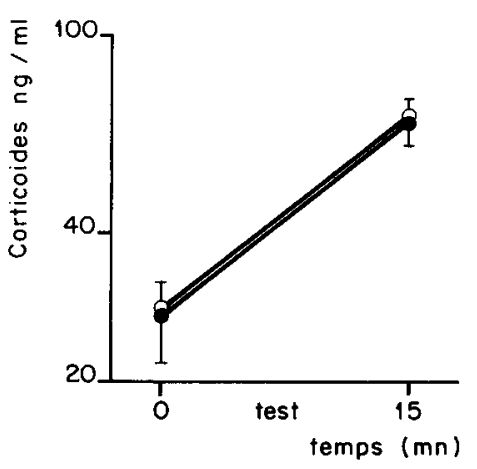

Corticoides

FIG. 2. - Taux d'ACTH ef de corticostéroïdes (moyenne \pm écart-type de la moyenne, $\mathrm{n}=8$ ) mesurés dans le plasma prélevé avant ef après le test de privation sociale réalisé à 7 semaines chez des porcelets sevrés à 3 semaines et d̀ 6 semaines. 
temps à approcher le récipient contenant de la nourriture que les animaux sevrés à 6 semaines (tabl. 1) ; la latence d'alimentation par contre ne varie pas de façon significative suivant le mode d'élevage. Les femelles ne diffèrent pas des mâles castrés ni dans le test de privation sociale ni dans le test de néophobie.

3) Test d'évitement. - Le nombre de réponses émises et le nombre de chocs reçus en évitemenł (fig. 3) ont été soumis à une analyse de variance non symétrique à 2 facteurs (4 séances $\times 4$ groupes expérimentaux, 4 à 7 répétitions). Dans chaque cas, la légitimité du modèle a été vérifiée par l'absence d'interaction. Le nombre de réponses augmente au fur et à mesure des séances $\left(\mathrm{F}_{7_{1}}^{3}=5,66 ; \mathrm{P}<0,01\right)$, tandis que le nombre de chocs reçus diminue $\left(F_{71}^{3}=7,35 ; P<0,001\right)$, témoignant de l'apprentissage de l'évitement continu. Les porcelets mâles castrés sevrés à 6 semaines ont des performances inférieures à celles des femelles sevrées à 6 semaines et à celles des animaux sevrés précocement $\left(F_{71}^{3}=4,73 ; P<0,01\right.$ pour le nombre de réponses ef $F_{71}^{3}=6,78 ; P<0,001$ pour le nombre de chocs reçus).

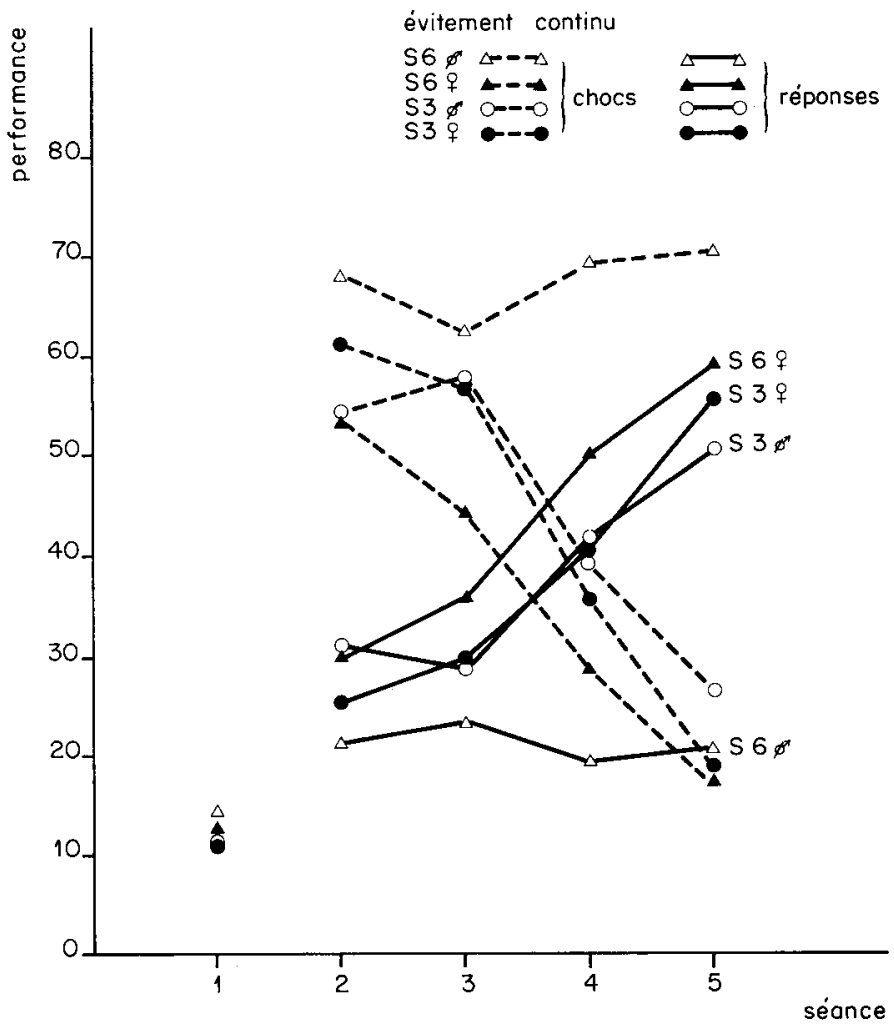

FIG. 3. - Performances en évitement continu des porcs sevrés à 3 semaines (S3) ef à 6 semaines (S6). En ordonnées figurent le nombre de chocs reçus et le nombre de réponses émises, pour chaque groupe expérimentàl, en fonction du sexe. En abscisses figure l'ordre des séances. La première séance correspond à l'exploration de la cage de test. 


\section{Discussion.}

Les porcelets sevrés précocément et élevés dans la loge de mise-bas (Fraser, 1978) ou dans des cages grillagées (Marx et Hoepfner, 1977a, b, c ; Marx et al., 1977 ; Van Putten et Dammers, 1976 ; Worsaae et Schmidt, 1980) présentent en règle générale une forte incidence de comportements anormaux (trépignements, massage avec le groin de l'abdomen des congénères, succion de la queve et des oreilles). Selon la plupart des auteurs, ces différences comportementales reflètent l'inconfort physique engendré par l'environnement voire la frustration consécutive à l'impossibilité d'exprimer certains comportements prédominants dans le répertoire naturel de l'espèce (fouille, tétée). Peu d'auteurs en ont recherché la traduction physiologique au niveau des systèmes hormonaux intervenant dans la réponse aux agressions. Chez des porcs sevrés à 3 semaines, Stanton et Mueller (1975) ont mis en évidence une augmentation importante et durable de l'activité des principales enzymes contrôlant le métabolisme des catécholamines ef de l'acétyl-choline au niveau de la médullo-surrénale et du ganglion cervical supérieur, témoignant d'une forte activation du système orthosympathique et médullo-surrénalien ; le contenu des surrénales en cortisol n'était toutefois pas modifié. Worsaae et Schmidt (1980) ont mesuré des taux plasmatiques de cortisol plus élevés chez des animaux sevrés précocement et élevés en cage grillagée.

Dans notre étude, le sevrage précoce a été accompagné d'une élévation transitoire de la cortisolémie (à J1), avec retour rapide aux taux de base. Par contre, le sevrage à 6 semaines dans la loge de mise-bas n'a pas eu d'influence notable sur l'activité cortico-surrénalienne et peut, de ce fait, être considéré comme moins stressant. L'activité corticosurrénalienne varie en fonction de l'âge : elle est maximale à la naissance, décroît rapidement au cours des 10 premiers jours puis plus lentement avant de se stabiliser au niveau caractéristique de l'âge adulte (Brenner et Gürtler, 1977 ; Dvorak, 1972). Les valeurs mesurées ici chez les porcelets en cage sont dans la zone de variation considérée comme normale pour des porcelets de 3 à 8 semaines. Par contre, les valeurs mesurées à 4 et 5 semaines chez les porcelets sous la mère apparaissent anormalement basses et ne rejoignent les valeurs aftendues qu'à la 6e semaine, avaní le sevrage. Les causes exactes de cette baisse de la cortisolémie (environnement, tétée, rythme d'activité...) sont difficiles à préciser, en raison de l'absence d'étude systématique des facteurs de variation de l'activité hypophyso-corticosurrénalienne chez le jeune.

Les deux modes d'élevage étudiés ici diffèrent par le niveau de stimulation extéroceptive et proprioceptive fourni par l'environnement. L'élevage en cage représente un environnement appauvri : les stimulations extéroceptives (bruit, lumière, contacts humains) sont peu intenses et toujours identiques; de plus, le faible espace au sol limite les mouvements et donc l'information sensorielle d'origine proprioceptive (récepteurs musculaires, articulaires et cutanés). A l'inverse, les porcelets laissés avec la truie dans la loge de maternité bénéficient d'un environnement diversifié, en raison du renouvellement quotidien de la litière, de la présence de la truie, de l'alternance jour/nuit et des nombreuses stimulations présentes dans un local non isolé. Deux types différents d'expérience se trouvent ainsi réalisés. Les épreuves les plus couramment utilisées pour mettre en évidence l'influence de l'expérience antérieure sur le comportement et la physiologie sont l'open-field, les mesures de néophobie et le comportement 
d'évitement (Ader, 1970 ; Denenberg, 1969 ; Levine et Otis, 1958 ; Levine et al., 1956, 1967 ; Weinberg ef al., 1978). Nous avons montré précédemment que ces tests appliqués au porc étaient sensibles, puisqu'ils permettent de distinguer des animaux d'origine génétique différente (Dantzer ef Mormède, 1978 ; Mormède et Dantzer, 1978). Pratiqué sur le porcelet, le fest d'open-field mesure surtout les réactions à la privation sociale (Dantzer, 1979) et les résultats obtenus laissent penser que les animaux sevrés à 6 semaines qui n'ont pas encore subi de perturbation sociale, sont plus sensibles à cette agression que les porcelets sevrés à 3 semaines et mélangés à d'autres animaux. Cette différence de sensibilité reste cependant mineure, puisqu'elle n'a pas de traduction hormonale. Une telle dissociation entre indices comportementaux et neuroendocriniens de la réactivité n'est pas exceptionnelle (Ader ef al., 1967 ; Hess et al., 1969 ; Stern ef al., 1973) et elle témoigne de la nécessité de considérer simultanément les deux types de réponse.

Dans l'épreuve de néophobie, l'augmentation de latence d'approche observée chez les porcelets sevrés à 3 semaines montre que ceux-ci ont une composante néophobique plus importante que les porcelets sevrés à 6 semaines, ce qui est certainement en rapport avec le faible niveau de stimulations auquel ils ont été soumis. La différence reste cependant faible et n'empêche pas les animaux d'utiliser l'information une fois celle-ci enregistrée, comme en témoigne l'absence de différences dans les latences d'alimentation.

Le mode d'élevage n'a pas d'influence par lui-même sur les performances en évitement actif. De fait, les résultats obtenus montrent l'existence d'une interaction entre le mode d'élevage et le sexe, la différence observée habituellement entre mâles castrés ef femelles (Dantzer et Mormède, 1978) disparaissant chez les animaux élevés en cage grillagée. L'absence de différences entre les sexes dans les autres épreuves, en accord avec nos résultats antérieurs, ne permet guère de spéculer sur les facteurs concourant à l'augmentation de performance des mâles castrés sevrés à 3 semaines. L'existence d'interactions complexes entre le type d'expérience antérieure, le sexe ef les caractéristiques de la situation test est un fait reconnu mais qui n'a pas encore reçu d'interprétation satisfaisante (Weinberg et Levine, 1977 ; Weinberg et al., 1978).

En conclusion, les différences observées dans les divers tests utilisés montrent que les influences à terme de l'élevage en cage grillagée sur la physiologie et le comportement du porcelet sont vraisemblablement minimes. Il conviendrait toutefois de préciser la signification de ce résultat par des expériences complémentaires visant à tester de façon systématique les conséquences de l'exposition à des stimulations standardisées sur le développement comportemental et physiologique du porcelet.

Reçu en décembre 1980.

Accepté en mars 1981.

Remerciements. - Nous remercions MM. Aumaitre, Cabello, Le Neindre, Signoret et Tillon pour leurs commentaires ef leurs suggestions sur les résultats de ce travail.

\section{Références}

ADER R., 1970. The effects of early experience on the adrenocortical response to different magnitudes of stimulation. Physiol. Behav., 5, 837-839. 
ADER R., FRIEDMAN S. B., GROTA L. J., 1967. "Emotionality » and adrenal cortical function : effects of strain, test and the 24-hour corticosterone rhythm. Anim. Behav., 15, 37-44.

BRENNER K. V., GÜRTLER H., 1977. Die Konzentration an Kortisol, Glukose und freien Fettsäuren im Blutplasma bei Schweinen in Abhängigkeit vom Alter sowie bei Sauen im Zeitraum der Geburt. Arch. exp. Vet. med. Leipzig, 31, 741-747.

DANTZER R., 1979. Intervention des endorphines dans le comportement émotionnel du porc. $C . R$. Acad. Sci. Paris, Sér. D, 289, 1299-1302.

DANTZER R., MORMĖDE P., 1978. Behavioural and pituitary-adrenal characteristics of pigs differing by their susceptibility to the malignant hyperthermia syndrome induced by halothane anesthesia. I. Behavioural measures. Ann. Rech. vét., 9, 559-567.

DANTZER R., MORMĖDE P., 1979. Le stress en élevage intensif, Masson, Paris, 128 pP.

DENENBERG V. H., 1969. The effects of early experiences, 95-130. In HAFEZ E. S. E., The behaviour of domestic animals. Baillière, Tindall \& Cassell, Londres, $2^{\mathrm{e}}$ éd.

DVORAK M., 1972. Adrenocortical function in foetal, neonatal and young pigs. J. Endocrinol., 54, 473-481.

FRASER D., 1974. The vocalizations and other behaviour of growing pigs in an "open-field 》 test. Appl. anim. Ethol., 1, 3-16.

FRASER D., 1978. Observations on the behavioural development of suckling and early-weaned piglets during the first six weeks after birth. Anim. Behav., 26, 22-30.

HESS J. L., DENENBERG V. H., ZARROW M. X., PFEIFER W. D., 1969. Modification of the corticosterone response curve as a function of handling in infancy. Physiol. Behav., 4, 109-111.

LEVINE S., OTIS L. S., 1958. The effects of handling before and after weaning on the resistance of albino rats to later deprivation. Can. J. Psychol., 12, 103-108.

LEVINE S., CHEVALIER J., KORCHIN S., 1956. The effects of early shock and handling on later avoidance learning. J. Personality, 24, 475-493.

LEVINE S., HALTMEYER G. C., KARAS G., DENENBERG V., 1967. Physiological and behavioral effects of infantile stimulation. Physiol. Behav., 2, 55-59.

MARX D., HOEPFNER G., 1977a. Verhalten frühabgesetzter Ferkel in Batteriekäfighaltung bei verschiedener Besatzdichte (Gegenüberstellung zum Verhalten von Saugferkeln). Disch. Tierärzll. Wschr., 84, 16-22.

MARX D., HOEPFNER G., 1977b. Verhalten frühabgesetzter Ferkel in Batteriekäfighaltung bei verschiedener Besatzdichtle (Gegenüberstellung zum Verhalten von Saugferkeln). Disch. Tierärztl. Wschr., 84, 45-51.

MARX D., HOEPFNER G., 1977c. Verhalten frühabgesetzter Ferkel in Käfiggruppenhaltung bei verschiedenen Flatdeckausführungen. 2. - Mitteilung : Gegenüberstellung zum Verhalten von Saufgerkeln und Ferkeln in Batteriekäfighaltung. Berl. Münch. Tierärztl. Wschr., 90, 358363.

MARX D., SCHRENK H. J., SCHMIDTBORN Chr., 1977. Spiel und Eliminationsverhalten von Saugferkeln und frühabgesetzten Ferkeln in Käfiggruppenhaltung. Disch. Tierärztl. Wschr., 84, 141-149.

MORMĖDE P., DANTZER R., 1978. Behavioural and pituitary-adrenal characteristics of pigs differing by their susceptibility to the malignant hyperthermia syndrome induced by halothane anesthesia. II. Pituitary adrenal function. Ann. Rech. vet., 9, 569-576.

STANTON H. C., MUELLER R. L., 1976. Sympathoadrenal neurochemistry and early weaning of swine. Amer. J. vet. Res., 37, 779-783.

STERN J. M., ERSKINE M. S., LEVINE S., 1973. Dissociation of open-field behavior and pituitaryadrenal function. Horm. Behav., 4, 149-162.

VAN PUTTEN G., DAMMERS J., 1976. A comparative study of the well being of piglets reared conventionally and in cages. Appl. anim. Ethol., 2, 339-356.

WEINBERG J., LEVINE S., 1977. Early handling influences on behavioral and physiological responses during active avoidance. Develop. Psychobiol., 10, 161-169.

WEINBERG J., SMOTHERMAN W. P., LEVINE S., 1978. Early handling effects on neophobia and conditioned taste aversion. Physiol. Behav., 20, 589-596.

WORSAAE H., SCHMIDT M., 1980. Plasma cortisol and behaviour in early weaned piglets. Acta vet. scand., 21, 640-657. 